\title{
Quantitative analysis of the correlation between preoperative cervical degeneration and postoperative heterotopic ossification after cervical disc replacement: minimum 10-year follow-up data
}

\author{
${ }^{*}$ Feifei Zhou, MD, ${ }^{1}$ Shuyang Li, MD, ${ }^{1}$ Yanbin Zhao, MD, ${ }^{1}$ Yilong Zhang, MD, ${ }^{1}$ Kevin L. Ju, MD, ${ }^{2}$ \\ Fengshan Zhang, MD, ${ }^{1}$ Shengfa Pan, MD, ${ }^{1}$ and Yu Sun, MD ${ }^{1}$ \\ 1Department of Orthopaedics, Peking University Third Hospital, Beijing, China; and ${ }^{2}$ Texas Back Institute, Plano, Texas
}

\begin{abstract}
OBJECTIVE The authors aimed to identify factors that may be useful for quantifying the amount of degenerative change in preoperative patients to identify ideal candidates for cervical disc replacement (CDR) in patients with a minimum of 10 years of follow-up data.
\end{abstract}

METHODS During the period from December 2003 to August 2008, 54 patients underwent CDR with a Bryan cervical disc prosthesis performed by the same group of surgeons, and all of the patients in this group with at least 10 years of follow-up data were enrolled in this retrospective analysis of cases. Postoperative bone formation was graded in radiographic images by using the McAfee classification for heterotopic ossification. Preoperative degeneration was evaluated in radiographs based on a quantitative scoring system. After univariate analysis, the authors performed multifactor logistic regression analysis to identify significant factors. To determine the cutoff points for the significant factors, a receiver operating characteristic (ROC) curve analysis was conducted.

RESULTS Study patients had a mean age of 43.6 years and an average follow-up period of 120.3 months. The patients as a group had a $68.2 \%$ overall incidence of bone formation. Based on univariate analysis results, data for patient sex, disc height, and the presence of anterior osteophytes and endplate sclerosis were included in the multivariate analysis. According to the analysis results, the identified independent risk factors for postoperative bone formation included disc height, the presence of anterior osteophytes, and endplate sclerosis, and according to a quantitative scoring system for degeneration of the cervical spine based on these variables, the ROC curve indicated that the optimal cutoff scores for these risk factors were $0.5,1.5$, and 1.5 , respectively.

CONCLUSIONS Among the patients who were followed up for at least 10 years after CDR, the incidence of postoperative bone formation was relatively high. The study results indicate that the degree of degeneration in the target level before surgery has a positive correlation with the incidence of postoperative ossification. Rigorous indication criteria for postoperative ossification should be applied in patients for whom CDR may be a treatment option.

https://thejns.org/doi/abs/10.3171/2020.4.SPINE191303

KEYWORDS postoperative heterotopic ossification; bone formation; osteophyte; follow-up; cervical

A $S$ a valid alternative for anterior cervical discectomy and fusion (ACDF), cervical disc replacement (CDR) may be used to treat cervical disc diseases and spondylosis that involve compression of the spinal cord or nerve root. Artificial disc replacement can maintain motion at the operative level ${ }^{1}$ and also provide adequate results that are equivalent to those for ACDF in aspects of neurological success and pain resolution. Un- like nonfusion approaches to spine surgery, in CDR surgery postoperative heterotopic ossification (HO) is considered a major complication. ${ }^{2,3}$ Through our experience with CDR, we found that the incidence of postoperative ossification was higher in patients with more severe preoperative cervical spondylosis. ${ }^{4}$ When the indications for cervical artificial disc placement were introduced, most of them were described through a qualitative approach but lacked

ABBREVIATIONS ACDF = anterior cervical discectomy and fusion; $\mathrm{AUC}=$ area under the curve; $\mathrm{CDR}=$ cervical disc replacement; $\mathrm{HO}=$ heterotopic ossification; $\mathrm{OALL}=$ ossification of the anterior longitudinal ligament; $R O C=$ receiver operating characteristic; $R O M=$ range of motion.

SUBMITTED November 1, 2019. ACCEPTED April 15, 2020.

INCLUDE WHEN CITING Published online July 17, 2020; DOI: 10.3171/2020.4.SPINE191303.

${ }^{*}$ F. Zhou, S. Li, and Y. Zhao contributed equally to this work. 
a quantitative standard. Using data from patients with a minimum of 10 years of follow-up, we sought to identify specific factors that are useful for quantifying preoperative degenerative changes in an effort to identify patients who are ideal candidates for CDR and thus minimize the incidence of postoperative $\mathrm{HO}$.

\section{Methods}

\section{Study Population and Data Collection}

We retrospectively identified 148 patients with a minimum of 10 years of follow-up data, all of whom underwent CDR with a Bryan cervical disc prosthesis at the same institution during the period from December 2003 to $\mathrm{Au}-$ gust 2008. Thirty-six patients who underwent surgery performed by surgeons not in our group and 58 patients who had incomplete imaging data at the follow-up examination were excluded from the total number. The 54 patients left were enrolled in the study. The indications for CDR were age 18-60 years, the presence of symptomatic radiculopathy or myelopathy caused by degenerative cervical discs between $\mathrm{C} 3$ and $\mathrm{C} 7$ that failed appropriate conservative treatment for a minimum of 6 weeks, and the absence of severe spondylosis, ossification of the posterior longitudinal ligament, and cervical instability (i.e., loss of disc height $>50 \%$, presence of bridging osteophytes, or $<2^{\circ}$ of segmental motion through the disc).

To identify predictive factors, relevant clinical findings, including age, sex, and the presence of ossification of the anterior longitudinal ligament (OALL), were collected from patient records. The target level was evaluated preoperatively on neutral lateral radiographs using a quantitative scoring system for the evaluation of cervical spine degeneration reported by Walraevens et al. that is based on 3 variables, disc height loss, anterior osteophyte formation, and the presence of endplate sclerosis. ${ }^{5}$ Evaluations of radiographic analysis results for all patients were conducted by one experienced surgeon. Disc height loss was determined as the target-level middle disc height compared with the normal middle disc height at an adjacent level. The anterior osteophyte length was measured with respect to the anteroposterior diameter of the corresponding vertebral body (measured at the middle of the vertebral body). The degree of endplate sclerosis was distinguished between sclerosis that was definite, just detectable, or not apparent. ${ }^{5}$ According to White's method, range of motion (ROM) before surgery and at final followup was evaluated through flexion-extension lateral radiographs. ${ }^{6}$ Postoperative ossification was graded using the McAfee classification for HO (Fig. 1). Radiographs of 2 patients in our study provide examples showing the classic image findings in patients with or without $\mathrm{HO}$ (Fig. 2).

The ethics committee of our hospital approved this study. Due to its retrospective design, the requirement for patient consent was waived.

\section{Statistical Analysis}

We performed statistical analysis with SPSS software (IBM Corp.). To describe the characteristics of this patient group, descriptive statistical analyses of primitive data sequences were conducted using a t-test for normally dis-
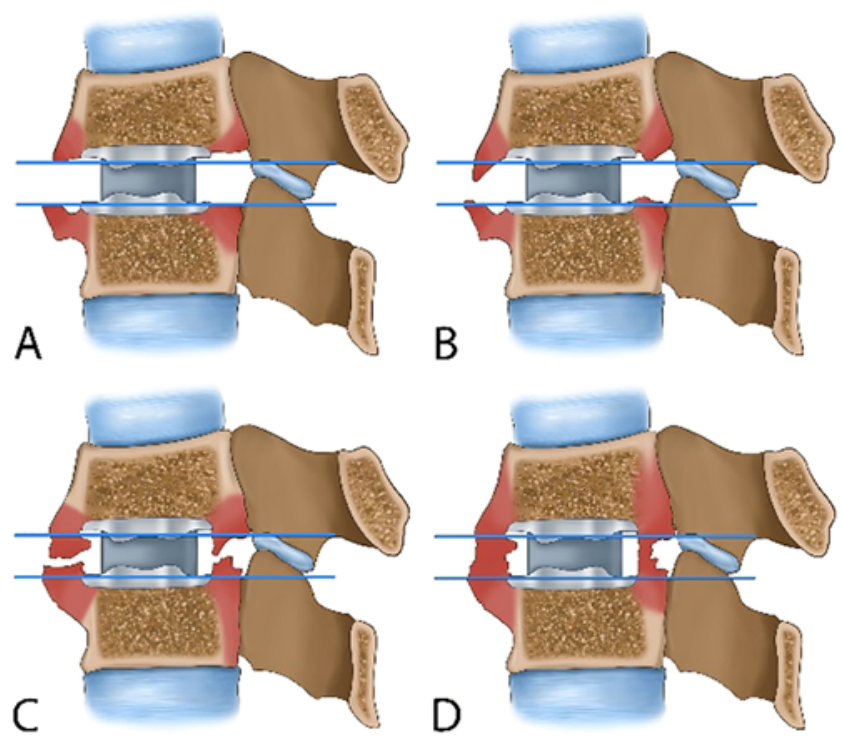

FIG. 1. McAfee classification of HO. A: Grade 1: bone formation detectable anterior to the vertebral body but not between the planes formed by the 2 vertebral endplates and not blocking motion. B: Grade 2: bone present between the planes formed by the vertebral endplates but not restricting motion. C: Grade 3: bone present between the planes formed by the vertebral endplates and restricting motion. D: Grade 4: complete bony ankylosis across the treated segment prevents motion through the prosthesis. Figure is available in color online only.

tributed variables, with standard deviations and means, to identify the differences associated with age and ROM between the 2 groups. We used a Mann-Whitney U-test if the data sets were abnormally distributed. To compare the categorical variables, Fisher's exact and chi-square tests were carried out, for which the analysis included disc height and the presence of OALL, anterior osteophytes, and endplate sclerosis. The factors identified as significant in the univariate analysis were then analyzed through logistic regression analysis. Receiver operating characteristic (ROC) curves were produced, and the results of the area under the curve (AUC) analysis $(>0.90$ and $\leq 1.00$ indicated excellent, $>0.80$ and $\leq 0.90$ indicated good, $>0.60$ and $\leq 0.80$ indicated fair, and $>0.50$ and $\leq 0.60$ indicated failure $)^{7}$ were used to identify the optimal cutoff points of the factors determined to be significant for evaluating the likelihood that CDR treatment will be effective for specific patients.

\section{Results \\ Patient Groups}

Our institution first performed cervical disc arthroplasty with a Bryan prosthesis in December 2003. In total, data for 54 patients were included in the study, all with a minimum of 10 years of follow-up (mean follow-up length 120.3 months), which comprised data for 66 disc replacement levels. At baseline, patients had a mean age of $43.6 \pm$ 7.1 years (46.3\% [25/54] female and 53.7\% [29/54] male). Of the 54 patients, 38 patients had cervical spondylotic myelopathy, 14 patients had cervical spondylotic radicu- 
Zhou et al.
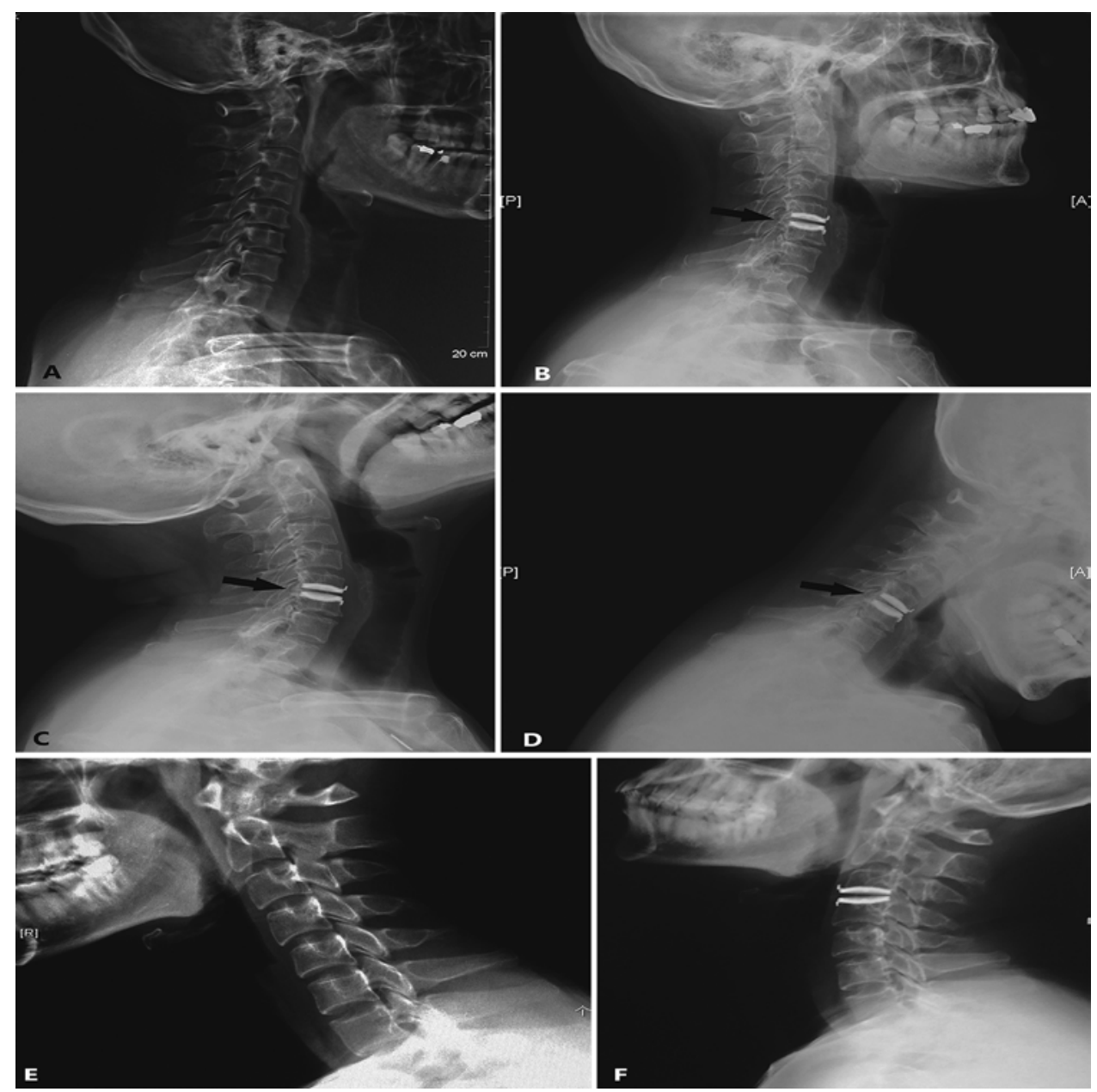

FIG. 2. A-D: Radiographs from one of 2 study patients. Preoperative neutral lateral radiograph $(A)$ and postoperative neutral (B), hyperextension (C), and hyperflexion (D) lateral radiographs at the 10-year follow-up, which showed grade $3 \mathrm{HO}$ at the surgical level (black arrows). E and F: Radiographs from the second patient. Preoperative neutral lateral radiograph (E) and postoperative neutral lateral radiograph at the 5-year follow-up (F), which showed no evidence of $\mathrm{HO}$.

lopathy, and 2 patients had cervical radiculopathy from a disc herniation. Regarding the surgical procedures performed, 43 patients underwent 1-level CDR, 10 patients underwent 2-level CDR, and 1 patient underwent 3-level $\mathrm{CDR}$. The demographic data for the patients and the surgical information are summarized in Table 1.

According to the evaluation of follow-up radiographs, $45(68.2 \%)$ of the 66 segments had developed HO at the surgical level at the time of the 10-year follow-up after CDR; 9 of 10 patients with 2 surgical levels showed HO in all levels, and 1 patient with 3 surgical levels showed $\mathrm{HO}$ in only 1 level. Of the 66 segments, according to the McAfee grades, $\mathrm{HO}$ was grade 2 in 6 segments, grade 3 in 18 segments, and grade 4 in 21 segments.

\section{Univariate Analysis}

The patients were divided into 2 groups based on whether any $\mathrm{HO}$ developed. The following 7 preoperative factors were evaluated: age, sex, preoperative ROM at the index level, disc height, anterior osteophytes at the index level, endplate sclerosis, and OALL.
TABLE 1. Baseline patient demographic data

\begin{tabular}{lc}
\hline & Value \\
\hline Age in yrs, mean (range) & $43.6(25-59)$ \\
\hline Sex & $29(53.7 \%)$ \\
\hline Male & $25(46.3 \%)$ \\
\hline Female & 43 \\
\hline CDR level & 10 \\
\hline 1 & 1 \\
\hline 2 & 38 \\
\hline 3 & 14 \\
\hline Cervical diagnosis & 2 \\
\hline Spondylotic myelopathy & \\
\hline Spondylotic radiculopathy
\end{tabular}

Values are presented as number of patients (\%) unless otherwise indicated. 
TABLE 2. Univariate analysis of preoperative factors

\begin{tabular}{|c|c|c|c|}
\hline & \multicolumn{3}{|c|}{ Non-HO } \\
\hline & HO Group & Group & p Value \\
\hline Age, mean (yrs) & 43.9 & 42.8 & 0.519 \\
\hline Sex, no. of segments & & & 0.009 \\
\hline Male & 29 & 6 & \\
\hline Female & 16 & 15 & \\
\hline Mean disc height score* & 1.53 & 0.38 & 0.000 \\
\hline Mean anterior osteophyte score & 1.64 & 0.62 & 0.000 \\
\hline Mean endplate sclerosis score ${ }^{*}$ & 1.69 & 0.90 & 0.000 \\
\hline OALL, no. of segments & & & 0.416 \\
\hline Yes & 6 & 1 & \\
\hline No & 39 & 20 & \\
\hline Preop ROM, ${ }^{\circ}$ & 7.82 & 9.14 & 0.130 \\
\hline
\end{tabular}

${ }^{*}$ Scores based on the degeneration evaluation scoring system described in the text.

Based on the results of univariate analysis, disc height, patient sex, and the presence of anterior osteophytes and endplate sclerosis were found to be statistically significant and thus were included for the subsequent multivariate analysis (Table 2).

\section{Multivariate Logistic Regression Analysis}

The multivariate logistic regression analysis included disc height, anterior osteophytes, endplate sclerosis, and patient sex, and disc height and the presence of anterior osteophytes and endplate sclerosis were identified as independent risk factors (Table 3 ). We then further analyzed these 3 factors with ROC curves. The AUCs calculated for the 3 significant factors were $0.845,0.868$, and 0.777 , respectively. ROC curve analysis showed that, based on the degeneration evaluation scoring system of the cervical spine, the optimal cutoff scores for disc height, anterior osteophytes, and endplate sclerosis were $0.5,1.5$, and 1.5 , respectively, with scores above the cutoff indicating a higher risk of HO, according to the ROC curve (Figs. 3-5).

\section{Discussion}

In recent years, the adoption of novel technologies such as CDR has sparked the development of not only new implantation techniques, but also novel approaches to the complications that can arise from the use of implantation devices. ${ }^{8,9}$ Several theoretical advantages of CDR compared with ACDF have been demonstrated. CDR can preserve motion in segments at the implantation site and reduce stress on the adjacent levels., ${ }^{3,810-15}$ However, the postoperative biomechanical changes after CDR are still not clearly understood. While CDR is typically associated with fewer complications than ACDF, HO can be a significant postoperative issue. The reported prevalence of $\mathrm{HO}$ following CDR varies widely depending on the study, which could reflect differences in patient demographics, length of follow-up, and different CDR designs. Regardless, most studies seem to agree that the prevalence of $\mathrm{HO}$ increases over time. ${ }^{16-20}$ Another study conducted by our institution found that except for loss of ROM, which was caused only by high-grade $\mathrm{HO}$, the appearance of $\mathrm{HO}$ did not worsen the clinical outcomes of these patients. ${ }^{27}$ Existing studies from other institutions also showed that there were no significant differences in terms of clinical outcomes, including scores for the Neck Disability Index and the SF-36 physical and mental components, between the $\mathrm{HO}$ and non-HO groups. ${ }^{26,28}$ The prevalence of postoperative bone formation at the long-term follow-up in our series of patients was relatively high $(36 / 54,66.7 \%)$. In our previous study, patients had a higher incidence of postoperative ossification if they had more severe preoperative spondylosis, indicating that postoperative ossification happened at the operative level, perhaps as a type of progressive bone formation in individuals who were already predisposed to forming bone rather than an $\mathrm{HO}$ occurring as a result of the surgery. ${ }^{4}$ Thus, appropriate patient selection is the crux of taking full advantage of the motion-preserving technology of CDR. However, most of the descriptions of indications for different cervical artificial prostheses are qualitative and lack specific quantitative criteria. Our study aimed to identify specific quantitative metrics preoperatively in order to minimize the incidence of $\mathrm{HO}$ postoperatively.

According to the quantitative scoring system for the evaluation of cervical spine degeneration published by Walraevens et al., ${ }^{5}$ disc height, anterior osteophytes, and endplate sclerosis are 3 factors used in their scoring system that were also all independent risk factors for postoperative $\mathrm{HO}$ development in our study. Yi et al. ${ }^{17}$ also suggested that sex and prothesis design affect the rate of HO. Moreover, researchers found there was a correlation between $\mathrm{HO}$ formation and advanced spine degeneration, but the correlation was not significant. Wu et al. ${ }^{21}$ also reported that patients with advanced degenerative changes demonstrated a higher rate of HO. Qi et al. ${ }^{22}$ evaluated patients undergoing CDR with the Discover Disc and determined that an increased T2 signal in the spinal cord, the number of operative levels, and the prosthesis to vertebral

TABLE 3. Multivariate logistic regression analysis of independent risk factors

\begin{tabular}{lcccccr}
\hline & $\beta$ & SE & Wald Test & df & p Value & $\operatorname{Exp}(\beta)$ \\
\hline Disc height & 3.035 & 1.207 & 6.319 & 1 & 0.012 & 20.802 \\
\hline Anterior osteophytes & 3.233 & 1.313 & 6.058 & 1 & 0.014 & 25.352 \\
\hline Endplate sclerosis & 3.630 & 1.498 & 5.872 & 1 & 0.015 & 37.698 \\
\hline Constant & -10.029 & 3.527 & 8.087 & 1 & 0.004 & 0.000 \\
\hline $\mathrm{df}=$ degrees of freedom; $\operatorname{Exp}(\beta)=$ odds ratio. & & & & &
\end{tabular}




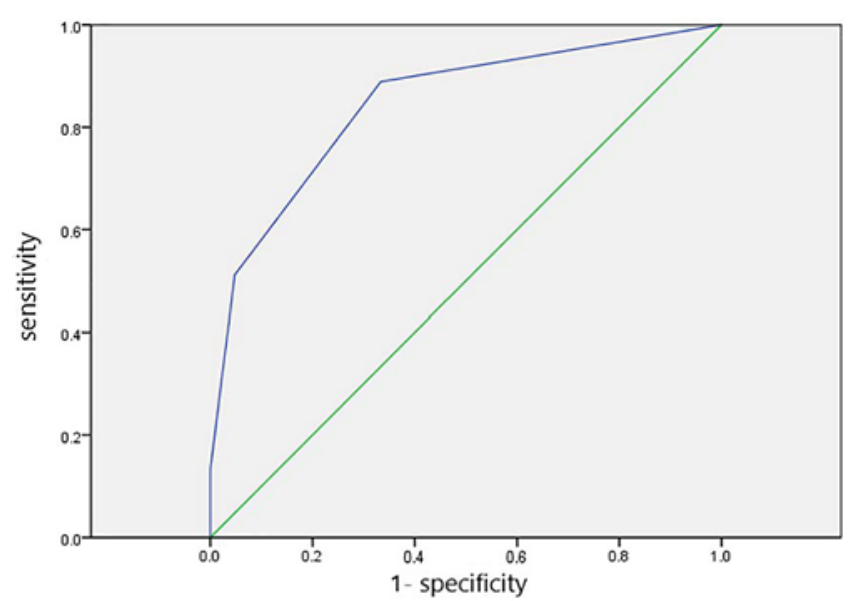

FIG. 3. ROC curve of disc height. Figure is available in color online only.

ratio were risk factors for $\mathrm{HO}$ development. Chung et al. ${ }^{10}$ suggested that preoperative uncovertebral hypertrophy was also one of the risk factors for $\mathrm{HO}$ development.

In contrast to other studies, our study focused on identifying predictive factors for quantifying the degree of preoperative spondylosis. Disc height loss was the most strongly predictive factor, with more than $50 \%$ height loss increasing the risk of postoperative $\mathrm{HO}$. The findings of Walraevens et al. ${ }^{5}$ were consistent with these results, that loss of disc height was the strongest predictive factor (4 out of a 9-point scale), followed by anterior osteophytes ( 3 out of a 9-point scale) and endplate sclerosis (2 out of a 9-point scale). The accuracy and simplicity of height loss measurement made it an optimal indicator for disc degeneration. Up to now, only a few studies, to our knowledge, have focused on the risk factors of postoperative HO development and determined specific indications for CDR based on quantitative rather than qualitative factors. Bertagnoli and $\mathrm{Kumar}^{23}$ proposed using a 4-mm disc height as one of the criteria for determining candidates for disc replacement, though the other factors used were still subjec-

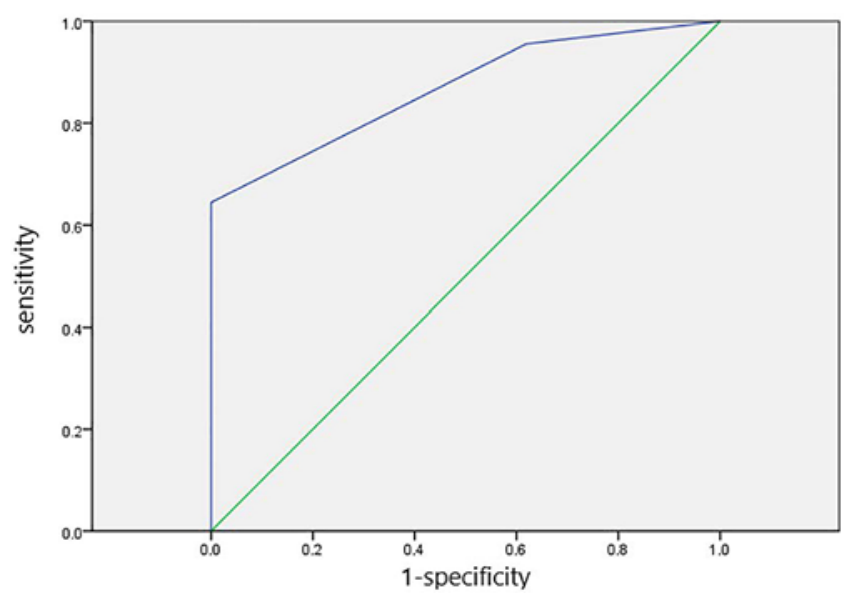

FIG. 4. ROC curve of anterior osteophytes. Figure is available in color online only.

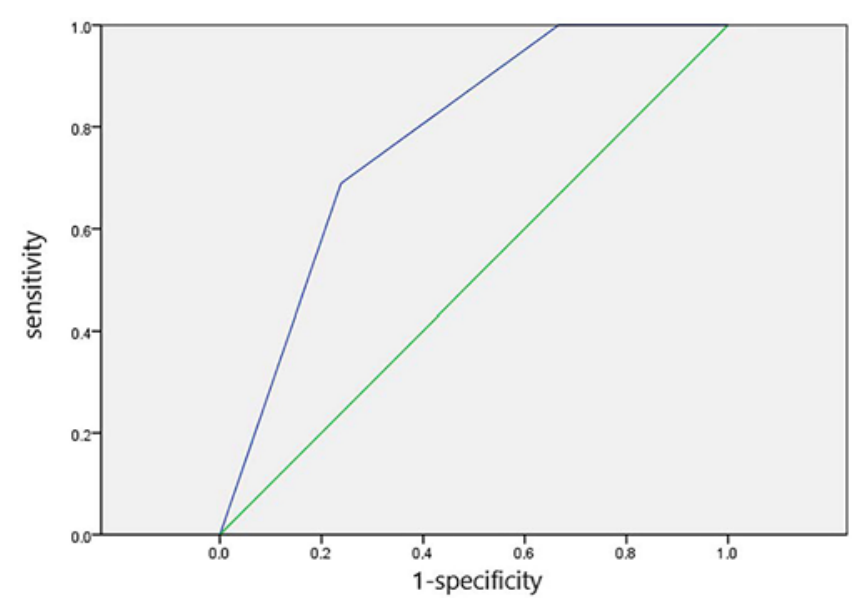

FIG. 5. ROC curve of endplate sclerosis. Figure is available in color online only.

tive and qualitative (i.e., degree of osteoarthritic changes of facet joints). When Auerbach et al. ${ }^{24}$ determined contraindications and postoperative complications of total disc replacement in an FDA investigational device exemption trial, disc height loss $>50 \%$ and anterior osteophytes were given as contraindications. Additionally, Jin et al. ${ }^{25}$ also showed through multivariate analysis that the presence of osteophytes is one of the risk factors of postoperative $\mathrm{HO}$ development. However, these studies did not propose specific numerical cutoffs for their qualitative criteria. Clearly, quantitative indications and contraindications for CDR can enable more objective determination of which patients will benefit the most from disc arthroplasty. Through the ROC curve analysis in this study, we obtained the AUC and cutoff values of disc height and the degree of anterior osteophytes and endplate sclerosis measurements that produce more objective indications that patients are suitable candidates for CDR surgery.

\section{Study Limitations}

This study has some limitations. The patient sample size was relatively small, and only one type of artificial disc was investigated, conditions that may have caused instability of the logistic regression equation and increased the risk of a type II error. To limit these risks, only a limited number of risk factors were evaluated, and this may be the reason why we could not screen risk factors other than cervical disc degeneration. Though the integrated mechanism of postoperative $\mathrm{HO}$ development occurring on the biomechanical level cannot be explained, we strongly recommend that patients with cervical disc degeneration, especially with disc height loss, undergo ACDF rather than CDR. Further analysis needs to be conducted to elucidate the significance and relationships between each of these possible predictors and other potential predictors.

\section{Conclusions}

Among the patients who were followed for more than 10 years after CDR surgery, the prevalence of postoperative bone formation after CDR was relatively high. 
However, patient selection, specifically how much of the preoperative degeneration happens at the surgical level, is a critical risk factor for the development of postoperative bone formation following CDR. Rigorous criteria, including nearly normal disc height and no more than mild indications of anterior osteophytes and endplate sclerosis, should be used when evaluating whether a CDR is the best treatment method for specific patients.

\section{References}

1. Dejaegher J, Walraevens J, van Loon J, et al. 10-year followup after implantation of the Bryan Cervical Disc Prosthesis. Eur Spine J. 2017;26(4):1191-1198.

2. Chen J, Wang X, Bai W, et al. Prevalence of heterotopic ossification after cervical total disc arthroplasty: a meta-analysis. Eur Spine J. 2012;21(4):674-680.

3. Kim KS, Heo DH. Do postoperative biomechanical changes induce heterotopic ossification after cervical arthroplasty?: a 5-year follow-up study. Clin Spine Surg. 2016;29(6):E309E313.

4. Zhou F, Ju KL, Zhao Y, et al. Progressive bone formation after cervical disc replacement: minimum of 5-year followup. Spine (Phila Pa 1976). 2018;43(3):E163-E170.

5. Walraevens J, Liu B, Meersschaert J, et al. Qualitative and quantitative assessment of degeneration of cervical intervertebral discs and facet joints. Eur Spine J. 2009;18(3):358-369. Published correction in Eur Spine J. 2009;18(3):370.

6. White AA III, Panjabi MM. The basic kinematics of the human spine. A review of past and current knowledge. Spine (Phila Pa 1976). 1978;3(1):12-20.

7. Hosmer DW, Lemeshow S. Assessing the fit of the model. In: Hosmer DW, Lemeshow S, eds. Applied Logistic Regression. 2nd ed. John Wiley \& Sons; 2000:143-202.

8. Park CK, Ryu KS. Are controversial issues in cervical total disc replacement resolved or unresolved?: a review of literature and recent updates. Asian Spine J. 2018;12(1):178-192.

9. Timothy J, Petralia V, Wilson JR. Use of an ultrasonic bone curet for the extraction of a cervical artificial disc: a novel application: a case report. JBJS Case Connect. 2018;8(1):e5.

10. Chung SB, Muradov JM, Lee SH, et al. Uncovertebral hypertrophy is a significant risk factor for the occurrence of heterotopic ossification after cervical disc replacement: survivorship analysis of Bryan disc for single-level cervical arthroplasty. Acta Neurochir (Wien). 2012;154(6):1017-1022.

11. Lazaro BC, Yucesoy K, Yuksel KZ, et al. Effect of arthroplasty design on cervical spine kinematics: analysis of the Bryan Disc, ProDisc-C, and Synergy disc. Neurosurg Focus. 2010;28(6):E6.

12. Pandey PK, Pawar I, Gupta J, Verma RR. Comparison of outcomes of single-level anterior cervical discectomy with fusion and single-level artificial cervical disc replacement for single-level cervical degenerative disc disease. Spine (Phila $\mathrm{Pa}$ 1976). 2017;42(1):E41-E49.

13. Rožanković M, Marasanov SM, Vukić M. Cervical disk replacement with discover versus fusion in a single-level cervical disk disease: a prospective single-center randomized trial with a minimum 2-year follow-up. Clin Spine Surg. 2017; 30(5):E515-E522.

14. Tian W, Yan K, Han X, et al. Comparison of the clinical and radiographic results between cervical artificial disk replacement and anterior cervical fusion: a 6-year prospective nonrandomized comparative study. Clin Spine Surg. 2017;30(5): E578-E586.

15. Yi S, Lee DY, Ahn PG, et al. Radiologically documented adjacent-segment degeneration after cervical arthroplasty: characteristics and review of cases. Surg Neurol. 2009;72(4): 325-329.
16. Mehren C, Heider F, Siepe CJ, et al. Clinical and radiological outcome at 10 years of follow-up after total cervical disc replacement. Eur Spine J. 2017;26(9):2441-2449.

17. Yi S, Shin DA, Kim KN, et al. The predisposing factors for the heterotopic ossification after cervical artificial disc replacement. Spine J. 2013;13(9):1048-1054.

18. Zeng J, Liu H, Chen H, et al. Comparison of heterotopic ossification after fixed- and mobile-core cervical disc arthroplasty. World Neurosurg. 2018;120:e1319-e1324.

19. Zeng J, Liu H, Chen H, et al. Effect of prosthesis width and depth on heterotopic ossification after cervical disc arthroplasty. Spine (Phila Pa 1976). 2019;44(9):624-628.

20. Zeng J, Liu H, Rong X, et al. Clinical and radiographic outcomes of cervical disc arthroplasty with Prestige-LP Disc: a minimum 6-year follow-up study. BMC Musculoskelet Disord. 2018;19(1):285.

21. Wu JC, Huang WC, Tu TH, et al. Differences between softdisc herniation and spondylosis in cervical arthroplasty: CTdocumented heterotopic ossification with minimum 2 years of follow-up. J Neurosurg Spine. 2012;16(2):163-171.

22. Qi M, Chen H, Cao P, et al. Incidence and risk factors analysis of heterotopic ossification after cervical disc replacement. Chin Med J (Engl). 2014;127(22):3871-3875.

23. Bertagnoli R, Kumar S. Indications for full prosthetic disc arthroplasty: a correlation of clinical outcome against a variety of indications. Eur Spine J. 2002;11(suppl 2):S131-S136.

24. Auerbach JD, Jones KJ, Fras CI, et al. The prevalence of indications and contraindications to cervical total disc replacement. Spine J. 2008;8(5):711-716.

25. Jin YJ, Park SB, Kim MJ, et al. An analysis of heterotopic ossification in cervical disc arthroplasty: a novel morphologic classification of an ossified mass. Spine J. 2013;13(4): 408-420.

26. Sundseth J, Jacobsen EA, Kolstad F, et al. Heterotopic ossification and clinical outcome in nonconstrained cervical arthroplasty 2 years after surgery: the Norwegian Cervical Arthroplasty Trial (NORCAT). Eur Spine J. 2016;25(7): 2271-2278.

27. Zhao Y, Zhang Y, Sun Y, et al. Application of cervical arthroplasty with Bryan cervical disc: 10-year follow-up results in China. Spine (Phila Pa 1976). 2016;41(2):111-115.

28. Zhou HH, Qu Y, Dong RP, et al. Does heterotopic ossification affect the outcomes of cervical total disc replacement? A meta-analysis. Spine (Phila Pa 1976). 2015;40(6):E332-E340.

\section{Disclosures}

The authors report no conflict of interest concerning the materials or methods used in this study or the findings specified in this paper.

\section{Author Contributions}

Conception and design: Sun. Acquisition of data: Zhao, Ju, Pan. Analysis and interpretation of data: Zhou, Li, Zhao, F Zhang. Drafting the article: Zhou, Li. Critically revising the article: all authors. Reviewed submitted version of manuscript: Sun, Zhou, $\mathrm{Li}, \mathrm{Y}$ Zhang. Approved the final version of the manuscript on behalf of all authors: Sun. Statistical analysis: Zhou, Li, Y Zhang, F Zhang. Administrative/technical/material support: Zhou, Li, Ju, Pan. Study supervision: Zhao.

\section{Correspondence}

Yu Sun: Peking University Third Hospital, Haidian District, Beijing, China. sunyuor@outlook.com. 